\title{
The Impact of Hot Melt Extrusion and Spray Drying on Mechanical Properties and Tableting Indices of Materials Used in Pharmaceutical Development
}

\author{
RAMAN IYER, ${ }^{1}$ SHRIDHAR HEGDE, ${ }^{1}$ YU-E ZHANG, ${ }^{1}$ JAMES DINUNZIO, ${ }^{1}$ DHARMENDRA SINGHAL, ${ }^{2}$ \\ A. MALICK, ${ }^{1}$ GREGORY AMIDON ${ }^{3}$
}

${ }^{1}$ Pharmaceutical \& Analytical R \& D, Hoffmann-La Roche Inc., Nutley, New Jersey, 07110

${ }^{2}$ Pharmaceutical Technical Development, F. Hoffmann-La-Roche, Ltd., Basel, Switzerland

${ }^{3}$ College of Pharmacy, University of Michigan, Ann Arbor, Michigan, 48109

Received 18 March 2013; revised 4 June 2013; accepted 17 June 2013

Published online 17 August 2013 in Wiley Online Library (wileyonlinelibrary.com). DOI 10.1002 /jps.23661

\begin{abstract}
The impact of melt extrusion (HME) and spray drying (SD) on mechanical properties of hypromellose acetate succinate (HPMCAS), copovidone, and their formulated blends was studied and compared with that of reference excipients. Tensile strength (TS), compression pressure $(\mathrm{CP})$, elastic modulus $(E)$, and dynamic hardness $\left(H_{\mathrm{d}}\right)$ were determined along with Hiestand indices using compacts prepared at a solid fraction of $\sim 0.85$. HPMCAS and copovidone exhibited lower $H_{\mathrm{d}}$, lower $\mathrm{CP}$, and lower $E$ than the reference excipients and moderate TS. HPMCAS was found to be highly brittle based on brittle fracture index values. The CP was $24 \%$ and $61 \%$ higher for HPMCAS after SD and HME, respectively, than for unprocessed material along with a higher $H_{\mathrm{d}}$. Furthermore, the TS of HPMCAS and copovidone decreased upon HME. Upon blending melt-extruded HPMCAS with plastic materials such as microcrystalline cellulose, the TS increased. These results suggest that SD and HME could impact reworkability by reducing deformation of materials and in case of $\mathrm{HME}$, likely by increasing density due to heating and shear stress in a screw extruder. A somewhat similar effect was observed for the dynamic binding index $\left(\mathrm{BI}_{\mathrm{d}}\right)$ of the excipients and formulated blends. Such data can be used to quantitate the impact of processing on mechanical properties of materials during tablet formulation development. @ 2013 Wiley Periodicals, Inc. and the American Pharmacists Association J Pharm Sci 102:3604-3613, 2013
\end{abstract}

Keywords: compaction; compression; excipients; formulation; tableting; hardness; extrusion; spray drying; mechanical properties; hiestand indices

\section{INTRODUCTION AND BACKGROUND}

The design and development of solid dosage forms and galenical processes rely on both the physicochemical and mechanical properties of the active ingredient, excipient components, and the mixtures thereof. Certainly, physical properties are closely linked to final product specifications such as purity, uniformity, dissolution, stability, appearance, and mechanical durability. ${ }^{1}$ While physical properties clearly influence powder flow and compression, the mechanical properties of materials, those properties of a material subject to an applied stress, are of great importance in solid dosage form development and manufacturing. The impact of such properties on material behavior during galenical processing has been demonstrated by instrumented tablet press, compression simulator, and mechanical testing devices. ${ }^{2-4}$

Based on the characteristics of the drug substance, excipients that complement or compensate for drug properties may be chosen along with processing equipments to favorably improve the behavior of materials under stress conditions. These evaluations are tied to FDA's new risk-based pharmaceutical quality assessment system in developing pharmaceutical cGMPs for the 21st century, where achieving and maintaining a state of control for a process is considered as beginning at the formula-

Correspondence to: Raman Iyer (Telephone: +732-560-8237; Fax: +973-2353769; E-mail: v4iyers@yahoo.com).

Journal of Pharmaceutical Sciences, Vol. 102, 3604-3613 (2013)

(C) 2013 Wiley Periodicals, Inc. and the American Pharmacists Association tion and process development phase and continuing throughout the commercial phase of a product's life cycle. This new assessment system focuses on pharmaceutical critical quality attributes such as pharmaceutical raw material properties as well as formulation and manufacturing processes as they relate to product performance. ${ }^{5-7}$ While there are some generalized procedures to evaluate material properties of active pharmaceutical ingredient (API), excipients, and mixtures, the impact of a given material (API or excipient) is dependent on its concentration in the formulation, its function, as well as any associated processing, and therefore needs to be studied in the context of its ability to affect the performance of a formulation or process.

The value of proper compaction characterization, i.e. assessment of the tensile strength (TS) - compression pressure (CP) solid fraction (SF) relationships leading to the compactability, tabletability, and compressibility (CTC) profiles, provides basic mechanical property information and has been discussed previously. $^{8}$ Along with TS, CP, and SF, the elastic modulus, permanent deformation pressure, and brittleness of compacts are additional important properties used to quantify the mechanical nature of materials. These properties, therefore, are of significant interest in supporting tablet development in a scientific manner. To characterize and compare these properties of materials with different deformation behavior, experimental methods and dimensionless tableting indices (DTI) were developed by Hiestand and Smith. ${ }^{9,10}$ The tableting indices are dimensionless numbers that profile the regions of interparticle 
contact, the so-called "isthmus" regions between contacting particles. It is at these regions where plastic deformation and the generation of large areas of true contact during decompression are necessary to produce significant tablet strength. The DTI, in combination with compaction characterization, yield a more complete assessment of the multidimensional nature of mechanical properties of materials. The bonding index (BI), for example, is a measure of the extent of plastic deformation or material strength that remains after decompression and recovery of a compact. It is thus a measure of bonding efficiency. A brittleness index called the brittle fracture index (BFI), derived from TS measurements, is a measure of brittleness (fracturing tendency) of compacted material. High BFI values are typically seen with brittle materials that undergo fragmentation. The third index called strain index (SI) indicates roughly the relative strain energy that could develop at a "crack" or other stressed region of the compact during elastic recovery after plastic deformation has occurred. This index combines the elastic and plastic deformation properties of a material and is a measure of the internal entropy or strain in a material when compacted. Finally, a fourth index termed the viscoelasticity index (VI) has been found to be useful as a measure of viscous (time dependent, irreversible) and elastic (reversible) deformation in a compact. 4

Since the tablet is the most prevalent dosage form that utilizes powder compression, the DTI developed by Hiestand has been used to characterize the behavior of materials during tableting. ${ }^{11-13}$ Statistical approaches using factorial designs have been employed to identify experimental conditions that can differentiate materials based on the tableting indices. These have included indenter specifications, compact size, and storage time, but the type of material (deformation behavior) has been the primary determinant of differentiation. ${ }^{14}$ The application of DTI to characterize powder compaction behavior by various methods has shown mixed results. The indices have been compared with that of Luenberger's percolation theory using different excipients with brittle or plastic characteristics. The comparison showed the two approaches to be complementary ${ }^{15}$ especially for plastically deforming excipients whereby the BI was found to increase with the characteristic relative density, $\rho^{*} r$, of such excipients. In empirical evaluations, the DTI were correlated with the impact of material properties on processing behavior such as granulation and capping or lamination during tableting. For example, in a study of the influence of the lubricant magnesium stearate on tableting indices of maltodextrins with serially increasing molecular weight, the TS, BI, and BFI were found to decrease with increasing level of magnesium stearate. ${ }^{16}$ This effect was more pronounced with the more plastic high molecular weight maltodextrin (M040) that exhibited almost 10-fold lower TS and fourfold lower BI when mixed with $0.48 \%$ magnesium stearate compared to virgin material without magnesium stearate. ${ }^{16}$ In a similar study on starches, ${ }^{17}$ the BFI was used to differentiate the tendency of various starches to ameliorate the brittle fracture of acetaminophen tablets during manufacturing. In a study correlating physical properties of drugs with processing behavior, a high BFI and low BI was seen with larger particle size, suggesting a tendency to cap or laminate during tableting. ${ }^{18}$ Moreover, higher BI and lower BFI was seen with wet granulated material compared to dry granulation, indicating a reduced potential for capping and the opportunity to achieve acceptable tablet strength using wet granulation. ${ }^{18}$
Amidon showed that specially crystallized lots of ibuprofen exhibited decreased powder flow properties with increasing BI in addition to reduced particle size and greater sphericity, indicating that the mechanical properties of a material also influence powder. ${ }^{3}$ The work of Wurster et al. demonstrated that estimating BI based on a linear function of composition was limited to binary mixtures of powders deforming in similar manner (plastic-plastic or brittle-brittle), whereas BI of blends of powders deforming in a dissimilar manner (brittle-plastic) seemed to exhibit a nonlinear relationship. ${ }^{19,20}$ The DTI did not predict behavior of new materials from a data set of indices of previously characterized materials using an artificial neural network approach, ${ }^{21}$ indicating the complex nature of predicting tablet properties for new materials. However, the nature of BI as a measure of bonding efficiency was shown in the characterization of microcrystalline cellulose (MCC) with varying moisture levels by Amidon and Houghton ${ }^{22}$ where both deformation pressure and TS were shown to decrease significantly with the moisture level greater than $5 \%$ as the excipient became more plasticized by adsorbed water, resulting in a relatively constant BI or bonding efficiency. This shows that materials with similar bonding indices may have different TS in proportion to their deformation hardness. It is also important to consider the experimental conditions used to generate DTI as they may impact the quantitative aspects and in turn, their predictive ability. In summary, mechanical property characterization and the development of predictive models is a complex function of the properties determined through CTC (i.e., TS CP, solid faction) and DTI (i.e., bonding, plastic deformation, brittleness, elasticity, viscoelasticity) methodologies. However, these measurements when combined provide greater insight into the mechanical properties of pharmaceutical materials and enhance our understanding of the impact of materials and processes on pharmaceutical materials.

Hot melt extrusion (HME) and spray drying are being increasingly employed to produce solid molecular dispersions of poorly soluble drugs, resulting in improved solubility and also to develop sustained, modified, and targeted drug delivery systems. ${ }^{23-27}$ During HME of pharmaceutical dosage forms, a blend of active ingredient, thermoplastic polymeric carrier, and other processing aids, including plasticizers and antioxidants, is heated and softened inside a screw extruder and then pressurized through a die into granules, cylinders, or films. ${ }^{24}$ The intense mixing and agitation imposed by the rotating screw cause deaggregation of suspended particles in the molten polymer resulting in a more uniform dispersion. ${ }^{23}$ In spray drying, the drug-polymer solution is atomized and dispersed into hot gas, which causes the solvent to evaporate and leads to the generation of spherical particles. ${ }^{27}$ Plasticizers are used to soften polymers and make them more flexible during melt extrusion. They decrease the glass transition temperature and the melt viscosity of a polymer by increasing the free volume between polymer chains and reducing their movement with respect to each other. ${ }^{23,28}$ While this improves the processing conditions (lower temperature and lesser torque, improved stability) during manufacturing of the extruded dosage form, it can also influence the physical and mechanical properties of the extrudate such as TS and elastic modulus. ${ }^{23,29}$ In drug-polymer solid dispersions, the small molecule compounds (drugs) in a polymer matrix can plasticize the polymer during HME, impacting its mechanical properties such as reduced TS of extruded films. ${ }^{30}$ Powders processed by HME are subjected not only to 
elevated processing temperatures but also to high pressure. The reduced free volume further retards molecular mobility and can prevent further densification during tableting, ${ }^{31,32}$ which could adversely impact product performance such as drug dissolution. Crowley et al. showed that guaifenesin matrix tablets containing binary mixtures of guaifenesin and ethyl cellulose, prepared by HME exhibited considerably slower drug release relative to those prepared by direct compression. ${ }^{33}$

While the improved dissolution rate of solid dispersions by HME and spray-drying processes is desirable, the mechanical properties of materials subject to these processes can impact their ability to form acceptable compacts during tablet development, often a desired end point in pharmaceutical formulation development.

\section{OBJECTIVES}

The purpose of the current study was to determine the mechanical properties of pharmaceutical excipients and their mixtures processed in different ways, focusing primarily on HME and spray drying. CTC profiles and DTI were employed in this study with the objective of developing an improved understanding of the impact of ingredient properties and processing on material behavior. The basic material properties of deformation pressure (i.e., dynamic hardness) and TS were measured at a fixed $\mathrm{SF}$, and these data were used to generate the tableting indices $\mathrm{BI}, \mathrm{BFI}, \mathrm{SI}$, and VI to gain greater insight into the mechanical properties of these materials than is possible from compaction characterization alone. The materials include a drug candidate (API) and formulated mixtures of API and excipients.

\section{MATERIALS AND METHODS}

Hypromellose acetate succinate (HPMCAS) (AQOAT LF grade; Shinetsu Corp., Japan), Copovidone (PVP VA64; BASF, Germany), MCC (Avicel PH 101; FMC Corp., Philadelphia, PA; Vivapur 102; JRS Pharma, Germany), Lactose (Pharmatose $350 \mathrm{M}$; DFE Pharma, Germany), dibasic calcium phosphate anhydrous or DCP-A (A-TAB; Innophos, Cranbury, NJ), croscarmellose sodium (AcDiSol; FMC Corp., Philadelphia, PA), colloidal silica (Aerosil 200; Evonik Degussa Corp., Germany), and magnesium stearate (Mallinkrodt Inc., St. Louis, MO) were purchased and used without further treatment with the exceptions of colloidal silica and magnesium stearate that were passed through a \# 40 and \# 30 mesh screen, respectively, prior to use. Compound A was provided by Chemical Synthesis at Hoffmann-La Roche Inc.

\section{Hot Melt Extrusion}

The excipients or blends were tested as received or processed in the following ways. HME was performed as follows: Copovidone was melt extruded in a Leistritz $18-\mathrm{mm}$ twin-screw extruder at $165^{\circ} \mathrm{C}$ at a maximum screw speed of $150 \mathrm{rpm}$. HPMCAS was melt extruded in a Haake Minilab at $175^{\circ} \mathrm{C}$ in a single screw configuration at a speed of $250 \mathrm{rpm}$. A dispersion of Compound A, referred to as dispersion HME, was prepared as follows: Copovidone, HPMCAS and Compound $\mathrm{A}$ in an approximate ratio of 0.3:0.5:0.2 were blended with $1 \%$ colloidal silica for 10 min, and the blend was extruded in a Leistritz 18-mm twinscrew extruder at $175^{\circ} \mathrm{C}$ at a screw speed of $150 \mathrm{rpm}$. A clear glassy extrudate was obtained in all cases. The extrudate in each trial was milled in a Fitzmill using a 0.033-in. mesh screen at $3500 \mathrm{rpm}$ or higher. A formulation designated as final blend HME was prepared by mixing the dispersion HME with MCC, croscarmellose sodium, colloidal silica, and crospovidone for 10 min at $22 \mathrm{rpm}$ in a Turbula blender. The blend was lubricated with magnesium stearate for $2 \mathrm{~min}$.

\section{Spray Drying}

A $5 \%$ solution of HPMCAS in acetone was spray-dried (Anhydro SD35; SPX Corporation, Charlotte, North Carolina) at 80$100^{\circ} \mathrm{C}$ inlet and outlet temperature of $65-75^{\circ} \mathrm{C}$. The material collected was used without further treatment.

\section{Preparation of Compacts}

Compacts of HPMCAS, copovidone, MCC, DCP-A, and lactose in an unprocessed ("as is") and processed (HME, SD) state were prepared over a range of SF (approximately $0.8-0.9$ ). In addition, compacts of mixtures of these excipients with or without Compound A were prepared. For each material, flat-face, square compacts of $3 / 4$-in. size and weighing approximately $4.5 \mathrm{~g}$ were prepared in a triaxial press at varying CP. Compacts were compressed and held at pressure for $90 \mathrm{~s}$ followed by a 120 -s linear decompression profile using computer control to maintain punch and die-wall pressure approximately equal. Compacts were stored over night at room temperature and $40 \% \mathrm{RH}$ prior to testing. Compacts were evaluated for SF and subjected to static and dynamic indentation and tensile testing as described below. The TS was determined based on the force necessary to cause tensile failure under transverse compression, analogous to the measurement of tablet crushing force or hardness.

\section{True Density Measurement}

The true density of all materials $\left(\mathrm{g} / \mathrm{cm}^{3}\right)$ was determined in duplicate by helium pycnometry (Micromeritics model 1330).

\section{Compact Testing}

The CP and TS were determined at several compact SF levels using quasistatic, out-of-die, tableting indices methods as described in earlier literature..$^{3,4,8,10,11} \mathrm{~A}$ specially designed a pendulum impact device, a quasistatic indentation tester, and a TS tester were used to measure the mechanical properties of compacts prepared over a SF range of $0.8-0.9$. The properties measured at each SF were CP, dynamic indentation hardness $\left(H_{\mathrm{d}}\right), \mathrm{TS}$, compromised tensile strength $\left(\mathrm{TS}_{\mathrm{o}}\right)$, and quasistatic indentation hardness $\left(H_{\mathrm{qs}}\right)$. In combination with these measured properties, the DTI of BI, BFI, SI, and VI were calculated using equations described in the literature. ${ }^{10}$ Since compact mechanical properties are extremely sensitive to the state of consolidation, it is critical to compare materials at the same SF. A standard reference state of consolidation of SF $=0.85$ is a practical target at which to compare materials. The mechanical property at a reference SF of 0.85 was used and reported here for comparison and estimated by interpolation from semilog linear regression analysis of measured mechanical property versus SF. For DCP-A, the mechanical properties were determined by interpolation at a SF of 0.65 since the standard SF range could not be achieved due to equipment limitations and extrapolation to $\mathrm{SF}=0.85$ was also performed for comparison purposes. 


\section{RESULTS AND DISCUSSION}

\section{True Density}

True density values typically range from 1.1 to $1.6 \mathrm{~g} / \mathrm{cm}^{3}$ range for organic crystalline materials and $2.0-2.9 \mathrm{~g} / \mathrm{cm}^{3}$ for inorganics. The true density measurements of all the organic materials ranged from 1.202 to $1.537 \mathrm{~g} / \mathrm{cm}^{3}$. The inorganic material, DCP-A, had a higher value of $2.83 \mathrm{~g} / \mathrm{cm}^{3}$.

\section{Mechanical Properties of "As Is" and Processed Excipients}

The mechanical properties of the materials tested are presented in Table 1. Several common excipients (MCC, lactose monohydrate, and DCP-A) are included for comparison with the excipients in "as is" and processed forms of interest in this study. HPMCAS and copovidone exhibited lower dynamic hardness, lower CP for compact formation than the reference excipients, and moderately high TS. In addition, HPMCAS was more elastic and brittle compared to the reference excipients. In comparison with other grades of lactose monohydrate (not shown), the lactose monohydrate tested in this study showed properties typical of lactose excipients but with somewhat higher TS values than that seen for some grades. MCC exhibited, as expected, very high TS and bonding properties (consistent with its generally accepted performance characteristics as a tableting excipient). DCP-A behaves as a typical inorganic material. It has a high density that requires very high $\mathrm{CP}$ to form a compact of moderate strength and has low elasticity (i.e., high Reduced modulus of Elasticity and low SI). None of the materials tested exhibited unusually high viscoelastic behavior (VI < 10).

\section{Compression Pressure}

The CP (punch force/punch area) is the pressure necessary to produce a densified compact defined by its SF. Desirable CP range from 40 to $125 \mathrm{MPa}$ for organic materials based on the ability of the powder to remain as a compact with desirable TS upon unloading as well as practical loading limits of tabletmanufacturing tooling. In comparison, DCP-A would require a very high $\mathrm{CP}$ to achieve a $\mathrm{SF}$ in this range and pharmaceutical formulations containing this excipient are typically compressed to lower SF s where an acceptable TS may be achieved. The tabletability profile (CP vs. SF) is known to be dependent on the process used to form compacts with compression speed being a well-known factor. ${ }^{8}$ Therefore, a direct comparison between measured CP in this study and CP obtained under different compression conditions such as a rotary tablet press is not appropriate ${ }^{8}$ since CP in this study reflects the pressure needed to form an isotropic, defect-free compact. However, a direct comparison among materials at the same SF prepared by the process outlined earlier may be done as is the case in this study. Among the materials tested, copovidone and HPMCAS exhibited compression forces on the low, but acceptable, end of the spectrum. These materials are therefore relatively soft, suggesting lower yield strength and ease of forming compacts at a SF of 0.85. MCC and lactose had $\mathrm{CP}$ in the typical range, whereas DCP-A would require a CP $>1000 \mathrm{MPa}$ (extrapolation to $\mathrm{SF}=0.85$ ), reflecting its high hardness and difficulty in reaching a $\mathrm{SF}$ of 0.85 under practical conditions of tablet compression.

For spray-dried and melt-extruded HPMCAS, the CP needed to achieve SF of 0.85 was $24 \%$ and $61 \%$ higher than that for "as is" material, respectively. This indicates that spray drying

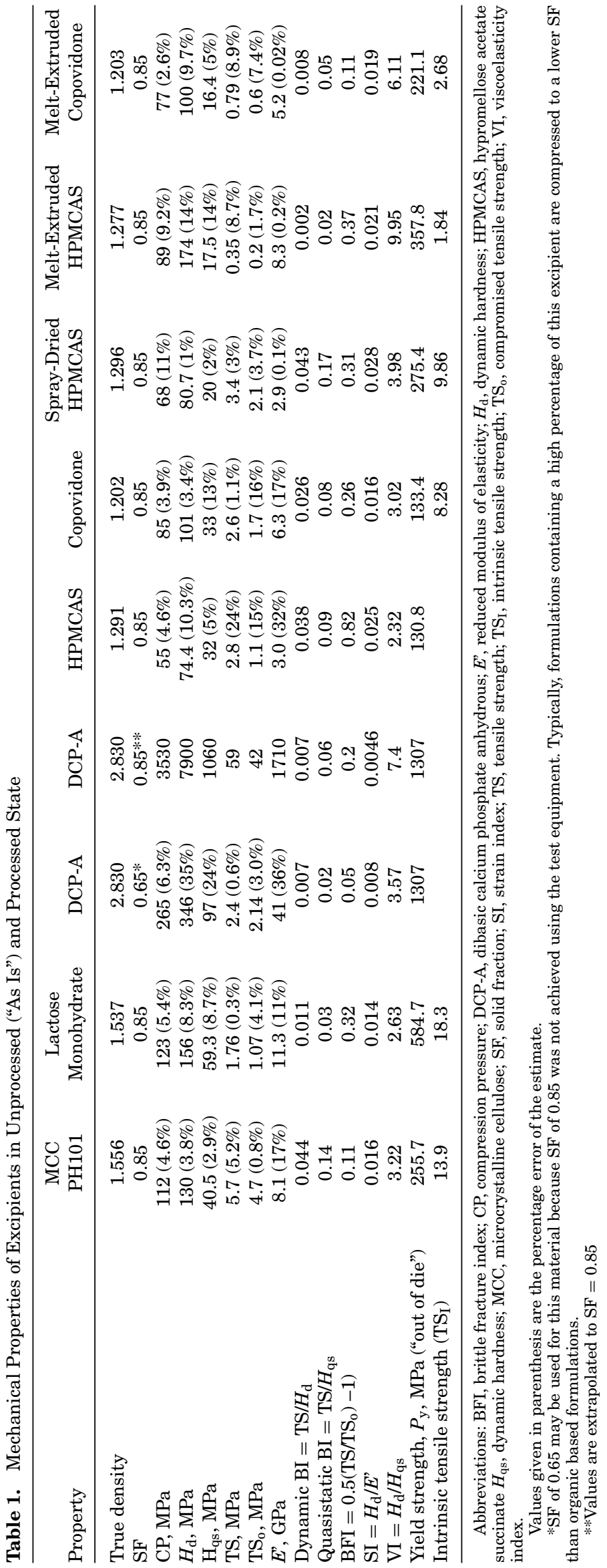



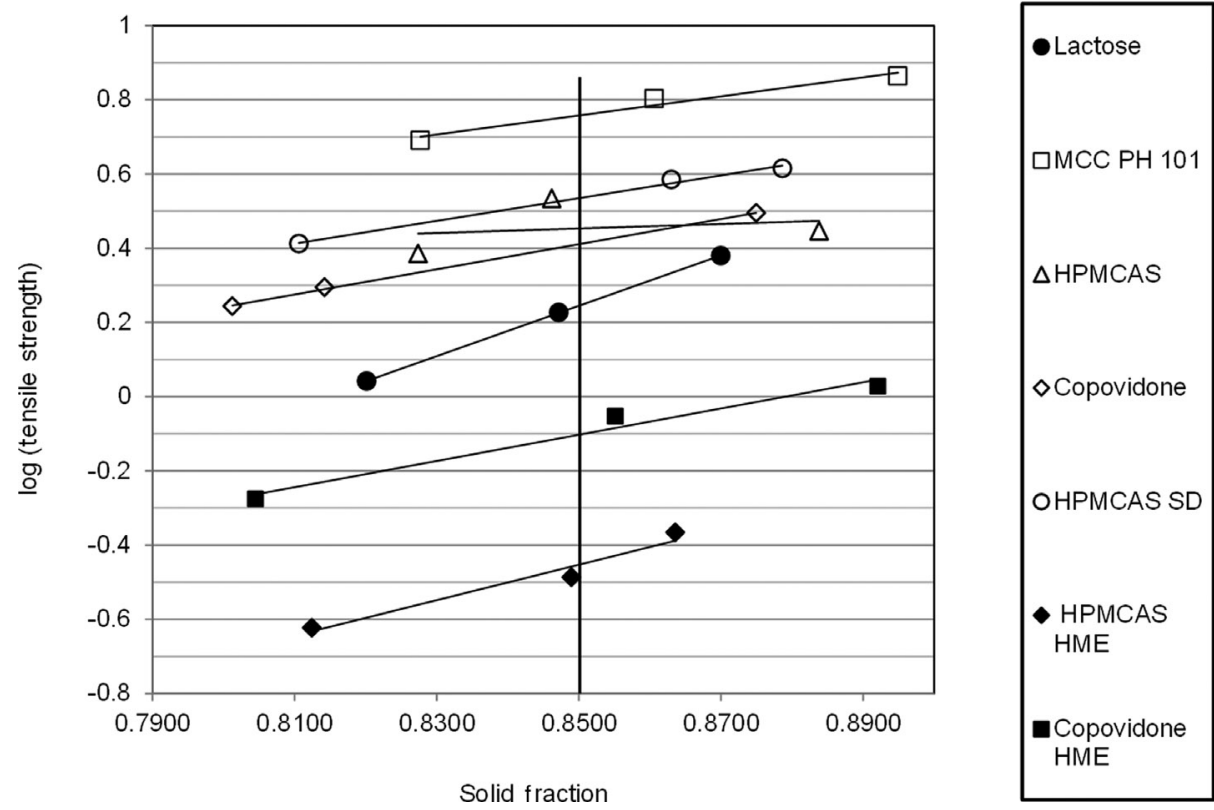

Figure 1. Compactibility (log TS vs. SF) curves for "as is" and processed excipients.

and melt extrusion reduced the ductility that manifested as a higher dynamic deformation pressure $\left(H_{\mathrm{d}}\right)$ for HPMCAS. By comparison, the $\mathrm{CP}$ of copovidone decreased by approximately $10 \%$ upon extrusion with no change in $H_{\mathrm{d}}$.

\section{Tensile Strength}

TS values of common organic excipients at a SF of 0.85 have been shown to range from a low of $0.5 \mathrm{MPa}$ to a high of $11 \mathrm{MPa}^{34}$ and a TS > $1 \mathrm{MPa}$ was achieved by all the "as is" materials tested in this study. As indicated in Table 1, lactose and MCC exhibited the lowest and highest TS among the "as is" materials tested whereas copovidone and HPMCAS had TS in the 2-3 $\mathrm{MPa}$ range, indicating that these materials form acceptable, strong compacts.

The relationship between TS and SF is referred to as "compactibility" of a material and is considered a predictor of tablet strength, independent of tableting speed, during formulation development. In the present study, compactibility of the materials was determined from tensile testing of compacts prepared at three different SF levels $>0.8$ except for DCP-A for which a SF close to this value could not be achieved within the normal CP range. A linear relationship of $\log$ (TS) versus SF was observed as shown in Figure 1 and is typical for various organic and inorganic materials including commonly used excipients such as lactose, starch, and cellulose as seen in the literature. ${ }^{8,35,36}$ $\mathrm{TS}$ at a $\mathrm{SF}$ of $0.85\left(\mathrm{TS}_{0.85}\right)$ was used for comparison among the materials.

In addition, the intrinsic strength at $\mathrm{SF}=1\left(\mathrm{TS}_{\mathrm{I}}\right)$ was extrapolated using linear regression of $\log (\mathrm{TS})$ versus SF plot for each material and presented in Table 1. MCC and lactose exhibited a greater $\mathrm{TS}_{0.85}$ and $\mathrm{TS}_{\mathrm{I}}$ that suggest a relatively high degree of deformation and bonding by either plastic and/or fracture mode, whereas HPMCAS and copovidone had lower $\mathrm{TS}_{\mathrm{I}}$. Upon HME, they exhibited even lower $\mathrm{TS}_{0.85}$ and $\mathrm{TS}_{\mathrm{I}}$, suggesting very poor ability to deform upon compression. However, the TS of HPMCAS increased upon spray drying as seen from Figure 2, indicating an enhanced ability to form strong compacts upon loading.

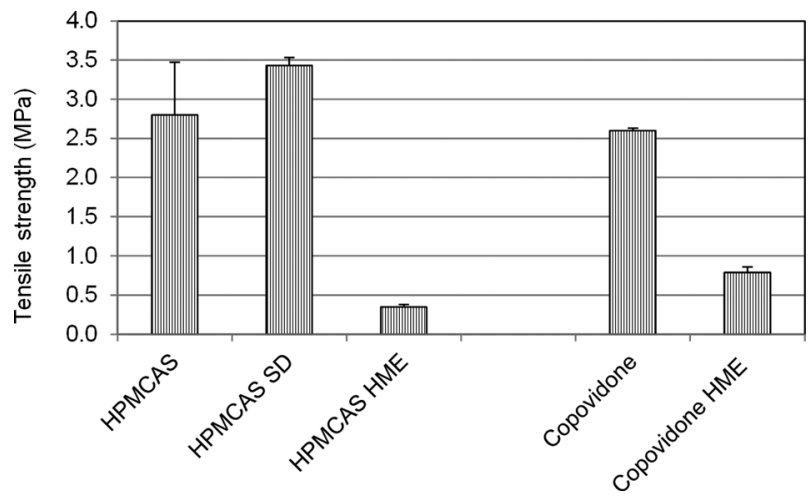

Figure 2. Tensile strength (TS \pm sd) of "as is" and processed HMPCAS and copovidone (sd, standard deviation).

For HPMCAS, an increase in pressure and particle density caused by heating and shear stress during melt extrusion in a screw extruder seems to have increased the strength of the milled HME particles significantly and diminished their ability to form strong bonds at points of contact. This suggests that, while melt extrusion can enable the formation of solid dispersions, the resultant extrudate may have a reduced ability to form strong compacts upon compression that is also reflected in the $\mathrm{TS}_{0.85}$.

\section{Hardness (e.g., Plasticity, Ductility)}

The dynamic hardness value $\left(H_{\mathrm{d}}\right)$ is an indication of the pressure required under dynamic, high-speed conditions to permanently deform a material. Material hardness (permanent deformation, hardness) is an important component of developing and maintaining compact strength because it allows for the formation and maintenance of true areas of contact between particles. Dynamic $H_{\mathrm{d}}$ values for organic tablet excipients, sometimes referred to as ductility or plasticity, typically range from 100 to $400 \mathrm{MPa}$ at a SF of $0.85 . .^{4,34}$ Based on this, soft or ductile 


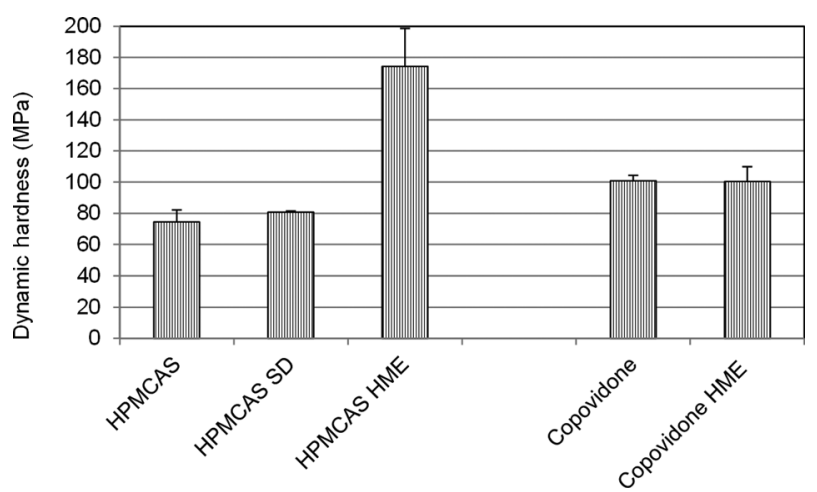

Figure 3. Dynamic hardness $\left(H_{\mathrm{d}} \pm \mathrm{sd}\right)$ of as is" and processed HPMCAS and copovidone.

materials may be considered to exhibit $H_{\mathrm{d}}$ in the range of 70 $100 \mathrm{MPa}$ whereas nonductile, hard materials may be considered to exhibit $H_{\mathrm{d}}>200 \mathrm{MPa}$. The hardness of the material is also measured under quasistatic (slow, $10 \mathrm{~min}$ ) indentation conditions $\left(H_{\mathrm{qs}}\right)$. The difference in these values is a measure of the viscoelasticity, or speed dependence of the material. The dynamic hardness values of the excipients tested are presented in Table 1 and were within the desirable range with the exception of HPMCAS, which behaves as a relatively soft and ductile material. This is also reflected in the low CP necessary to compact this material to a SF of 0.85 . DCP-A had a high dynamic hardness value even at a low $\mathrm{SF}$ of 0.65 , indicating that it is a very hard material.

The dynamic hardness of HPMCAS was significantly increased upon melt extrusion, as seen from Figure 3, likely due to the increase in material density and a possible change in the polymeric orientation with no significant changes in the chemical composition of the polymer. ${ }^{37}$ Since hard materials are difficult to mill and compact, this aspect of impact of melt extrusion on hardness of HMPCAS must be noted. Dynamic hardness of copovidone remained unchanged upon melt extrusion.

\section{Reduced Modulus (Elasticity)}

The reduced modulus of elasticity $\left(E^{\prime}\right)$ is a measure of the elastic property of a material. While not the same as the elastic modulus, $E$, it is generally proportional to $E$. A relatively low value of $E$ ' generally reflects a rather elastic material, whereas a large $E$ ' reflects a material that is relatively stiff. HPMCAS had the lowest $E$ ' and was the most elastic of the materials tested followed by copovidone. DCP-A had a very high $E$ ' that indicates it is a highly inelastic material.

\section{Tableting Indices}

\section{Brittle Fracture Index}

The BFI is a measure of the brittleness of a compact and indicates the mechanism of stress relief between particle bonds during tablet decompression. The stress relief mechanism occurs either by plastic deformation or bond fracture within particles. The BFI thus reflects the sensitivity of a compact to macroscopic flaws. The BFI is scaled to range between 0 for nonbrittle materials to 1 for very brittle or glassy materials. BFI values below 0.3 are generally desirable for tablet formulations. A BFI > 0.5 indicates that the material is quite brittle.

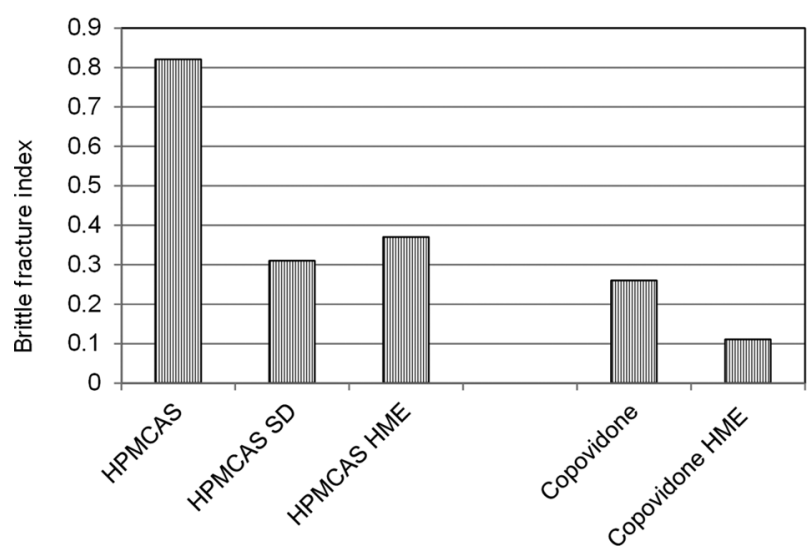

Figure 4. Brittle fracture index of "as is" and coprocessed excipients.

The BFI of HPMCAS was very high as seen from Table 1, indicating the brittle nature of compacts formed from this material. In comparison, copovidone exhibited only moderately low brittleness (BFI $=0.26)$ similar to that of lactose whereas MCC was the least brittle.

Upon spray drying and melt extrusion, the BFI decreased for both HPMCAS and copovidone, as shown in Figure 4. The BFI values were $0.3-0.4$ for HPMCAS SD and HME and $\sim 0.1$ for copovidone HME. This suggests that processes such as spray drying and melt extrusion can lower the brittleness of materials.

\section{Bonding Index}

The BI is a measure of the survival of tablet strength following decompression. It is therefore a measure of bonding efficiency and characterizes the tendency of the material to remain intact after tableting. It is determined under dynamic conditions $\left(\mathrm{BI}_{\mathrm{d}}\right)$ representative of high-speed compression and under quasistatic $\left(\mathrm{BI}_{\mathrm{qs}}\right)$ or slow compression conditions. At maximum $\mathrm{CP}$, the bonded areas are at a maximum because the true areas of contact are maximized. During decompression, some of that area is "lost" due to elastic recovery, additional plastic deformation, and/or fracture. A high BI suggests that a relatively larger proportion of the strength formed during compression remains intact after decompression. Typical values of $\mathrm{BI}_{\mathrm{d}}$ range from 0.002 for excipients that are considered to be weakly bonding to 0.03 or greater for excipients that exhibit strong bonding ability (e.g., MCC). ${ }^{4,34}$ The $\mathrm{BI}_{\mathrm{d}}$ of copovidone (0.026) and HPMCAS (0.038) was higher than that of lactose (0.011) (Table 1), indicating that during the compression-decompression process, a large fraction of the true area of contact is maintained for these two materials and that they are relatively efficient in their bonding properties. MCC, given its highly plastic deformation, had the highest $\mathrm{BI}_{d}$ value (0.044). In comparison, DCP-A has a low BI, which reflects the need for a high $\mathrm{CP}$ to form compacts of moderate strength. It is worth pointing out, however, that despite the low BI of DCP-A, it is still an acceptable excipient for tablet development since tablets with acceptable strength can be achieved using practical CP.

The $\mathrm{BI}_{\mathrm{d}}$ decreased significantly for both HPMCAS and copovidone upon melt extrusion, as seen from Figure 5. This suggests that the decrease in BFI upon melt extrusion of these excipients, though desirable, did not translate into an appreciable increase in plasticity. Since $\mathrm{BI}_{\mathrm{d}}$ is a ratio of TS to dynamic 


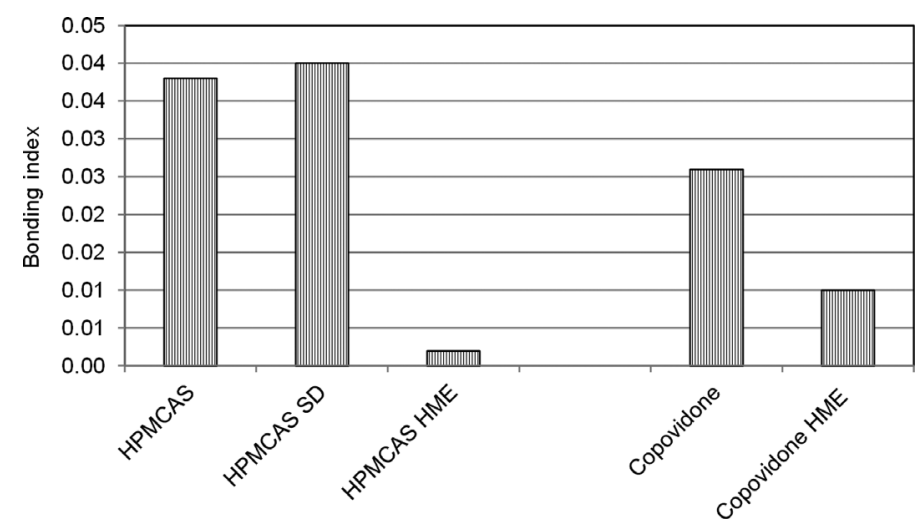

Figure 5. Dynamic bonding index of "as is" and coprocessed excipients.

hardness, a decrease in TS or increase in dynamic hardness can lower $\mathrm{BI}_{\mathrm{d}}$. For both excipients, upon melt extrusion, the TS decreased significantly while dynamic hardness remained the same for copovidone but increased for HPMCAS. The decrease in $\mathrm{BI}_{\mathrm{d}}$ with melt extrusion was more significant for HPMCAS. The practical implication of this observation is that it would require higher CP to prepare compacts of melt-extruded HPMCAS with acceptable hardness and therefore formulation of tablets containing melt-extruded HPMCAS may require use of materials that have higher $\mathrm{BI}_{\mathrm{d}}$ such as MCC or even HPMCAS in "as is" state. As stated earlier, the process of melt extrusion is essentially a densification process as the materials are subject to a high pressure resulting in a higher dynamic hardness. Upon milling the extrudate, the hard particles exhibit poor bonding and lower TS, resulting in a CP $61 \%$ greater than for "as is" material to achieve a SF of 0.85 . This is similar to the finding on the poor compressibility of melt-extruded particles. ${ }^{32}$ Spray-dried HPMCAS had a higher BI than "as is" material.

The impact of dynamic hardness (or ductility, $H_{\mathrm{d}}$ ) on CP needed to achieve SF of 0.85 is shown in Figure 6 . With an increase in $H_{\mathrm{d}}$, the $\mathrm{CP}$ to achieve $\mathrm{SF}$ of 0.85 was greater as expected since the deformation properties of a material would be expected to influence the $\mathrm{CP}$ needed to form a compact. A

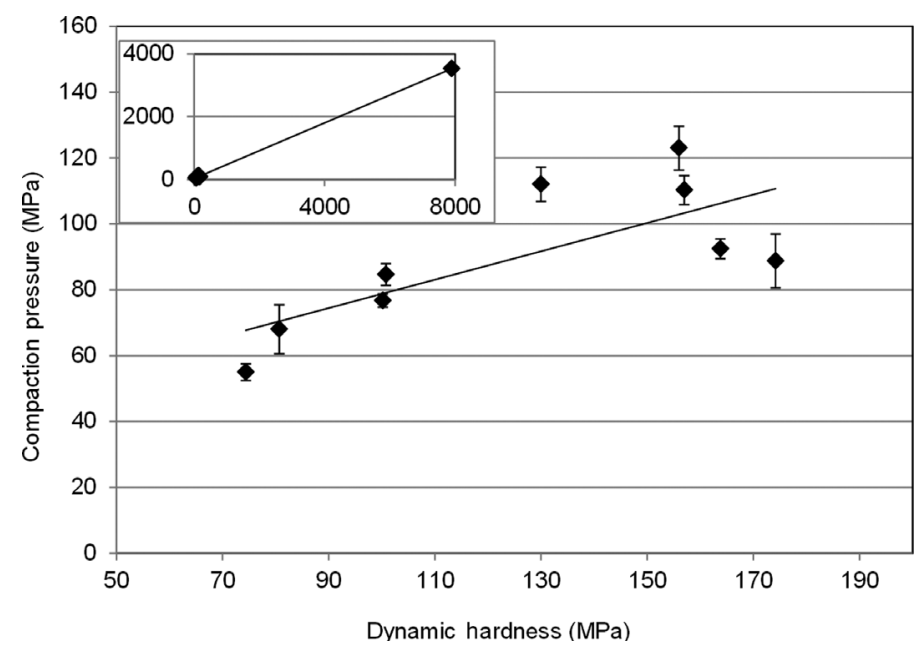

Figure 6. Effect of $H_{\mathrm{d}}$ of excipients on $\mathrm{CP}(\mathrm{CP} \pm \mathrm{sd})$ to achieve $\mathrm{SF}$ of 0.85 (Inset: includes DCP-A data at SF of 0.85). linear regression of the data in Figure 6 was performed without including the DCP-A values. Using the regression equation $\left(r^{2}=0.5531\right)$, the CP for DCP-A at $H_{\mathrm{d}}$ of $7900 \mathrm{MPa}$, representing a $\mathrm{SF}$ of 0.85 , was predicted at $3438.2 \mathrm{MPa}$ whereas the observed $\mathrm{CP}$ was $3530 \mathrm{MPa}$. This indicates good agreement between the two values, and that the relationship between $H_{\mathrm{d}}$ and $\mathrm{CP}$ is quite linear given the limited data set for the materials tested. The inset in Figure 6 shows the regression line including experimental DCP-A data.

\section{Strain Index}

The SI is a measure of the elasticity of a compact material. A material with a high SI will likely exhibit higher elastic recovery after compression. In comparing the materials tested, HPMCAS was found to exhibit a relatively higher SI, indicating its greater elasticity than other materials. In addition, the SI was higher for spray-dried and melt-extruded HPMCAS. The higher elasticity can also lead to a lower TS of the compact, which may further explain the lower $\mathrm{BI}_{d}$ for melt-extruded HPMCAS. This is also reflected in the reduced modulus of elasticity mentioned earlier. The SI of copovidone, lactose, and MCC was similar. The SI of DCP-A was very low, indicating its lack of elasticity.

\section{Viscoelasticity}

A comparison of the dynamic $\left(H_{\mathrm{d}}\right)$ and quasistatic hardness $\left(H_{\mathrm{qs}}\right)$ values can be used to assess the viscoelastic $(\mathrm{VE})$ nature of a material. Viscoelasticity is a kinetic phenomenon that refers to a material's ability to rapidly relieve stresses during powder compression. Viscoelasticity values in the range $2-10$ are noted in Table 1 are commonly observed for pharmaceutical excipients and reflect a minimal (e.g., typical) dependence of pharmaceutical material properties on the rate of loading during compact formation or tablet press speed. ${ }^{4}$ For some polymeric materials, the viscoelasticity value can approach 1000 or more (e.g., pregelatinized starch), indicating a significant dependence of material deformation on the rate of loading (or press speed) and time of storage effects. All the materials tested in this study exhibited VE in the range of $2-10$, suggesting the dependence of compact strength on press speed would be similar to excipients such as lactose and MCC. However, the relatively higher values for melt-extruded HPMCAS suggest a potentially greater dependence on the rate of loading or press speed.

\section{Mechanical Properties of Formulations of Compound A}

The mechanical properties of formulations containing Compound A were also evaluated. The TS of melt-extruded copovidone, HMCAS, dispersion HME, and final blend HME is shown in Figure 7. The TS varied in the order of HPMCAS HME < copovidone HME $<$ dispersion HME $<$ final blend HME. The inclusion of Compound A and copovidone in the dispersion HME increased the TS and adding MCC to form the final blend HME further increased the TS compared to dispersion HME alone.

The $\mathrm{BI}_{\mathrm{d}}$ of dispersion HME was intermediate to that of copovidone and HPMCAS HME, which indicates an associative mixture effect whereby addition of copovidone to HPMCAS increases its $\mathrm{BI}_{\mathrm{d}}$. Upon addition of plastically deforming MCC to the dispersion HME, the $\mathrm{BI}_{d}$ of final blend HME increases, reaching a value of 0.008 , similar to that of copovidone, as shown in Figure 8. 


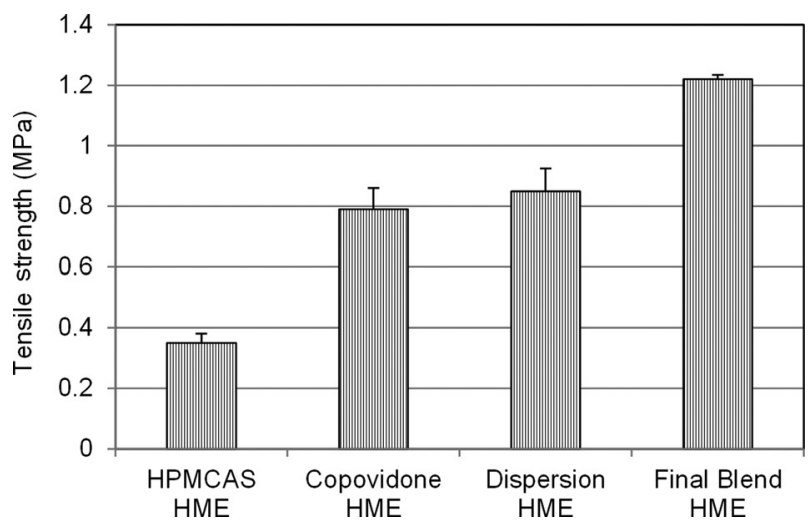

Figure 7. Tensile strength ( $\mathrm{TS} \pm \mathrm{sd}$ ) of melt extruded excipients and their mixtures with Compound A.

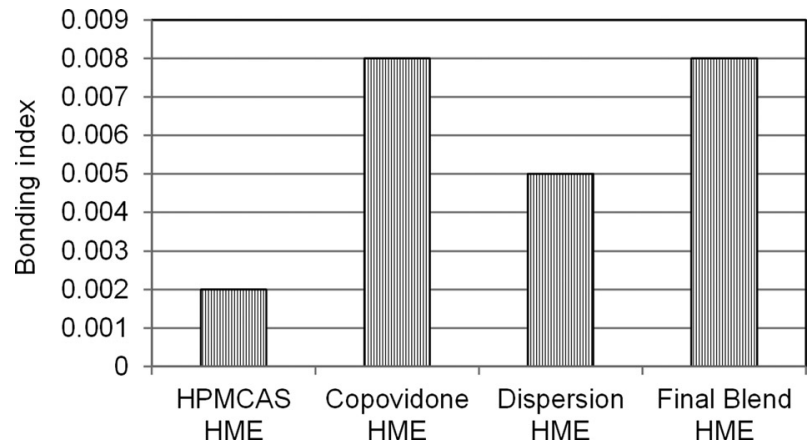

Figure 8. Dynamic bonding index of melt extruded excipients and their mixtures with Compound A.

In the present study, it was observed that when materials with different deformation behaviors are mixed in different ratios, the resultant mixtures exhibited a composite deformation behavior that was intermediate to that of the individual components. These results demonstrate the ability of the compaction

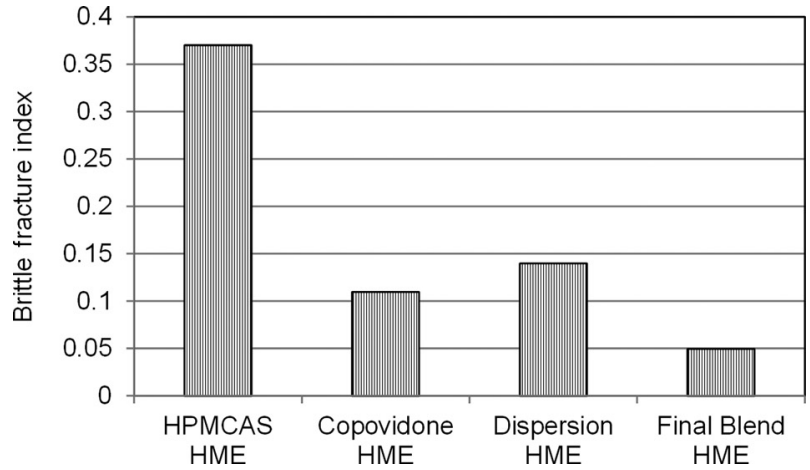

Figure 10. Britle fracture index of melt-extruded excipients and their mixtures with Compound A.

data and tableting indices to quantitate the impact of API and excipients on intermediate and final product properties.

The impact of the addition of HPMCAS to copovidone during melt extrusion on the TS of tablets prepared from milled extrudate is shown in Figure 9. It is seen that with increasing amount of HPMCAS up to $80 \%$, the tablet TS is reduced up to three- to fourfold over a compression force range of 12$33 \mathrm{kN}$. These data are also consistent with the conclusion that the properties of mixtures exhibit a composite behavior that is intermediate and roughly additive in proportion to their respective volume fractions, to that of the individual components. The addition of plastically deforming materials such as MCC is therefore effective in compensating for the $\mathrm{BI}_{\mathrm{d}}$ lowering effect of HPMCAS and predictable based on the results of Figure 9.

The BFI for formulations of Compound $\mathrm{A}$ is shown in Figure 10. The high BFI of HPMCAS HME extrudate in its glassy state suggests a lack of significant bonding contacts among the particles resulting in low TS of compacts. The lowering of BFI upon addition of copovidone and MCC is likely due to the resultant significant plastic deformation of these excipients leading to a higher compact TS and reduced brittleness.

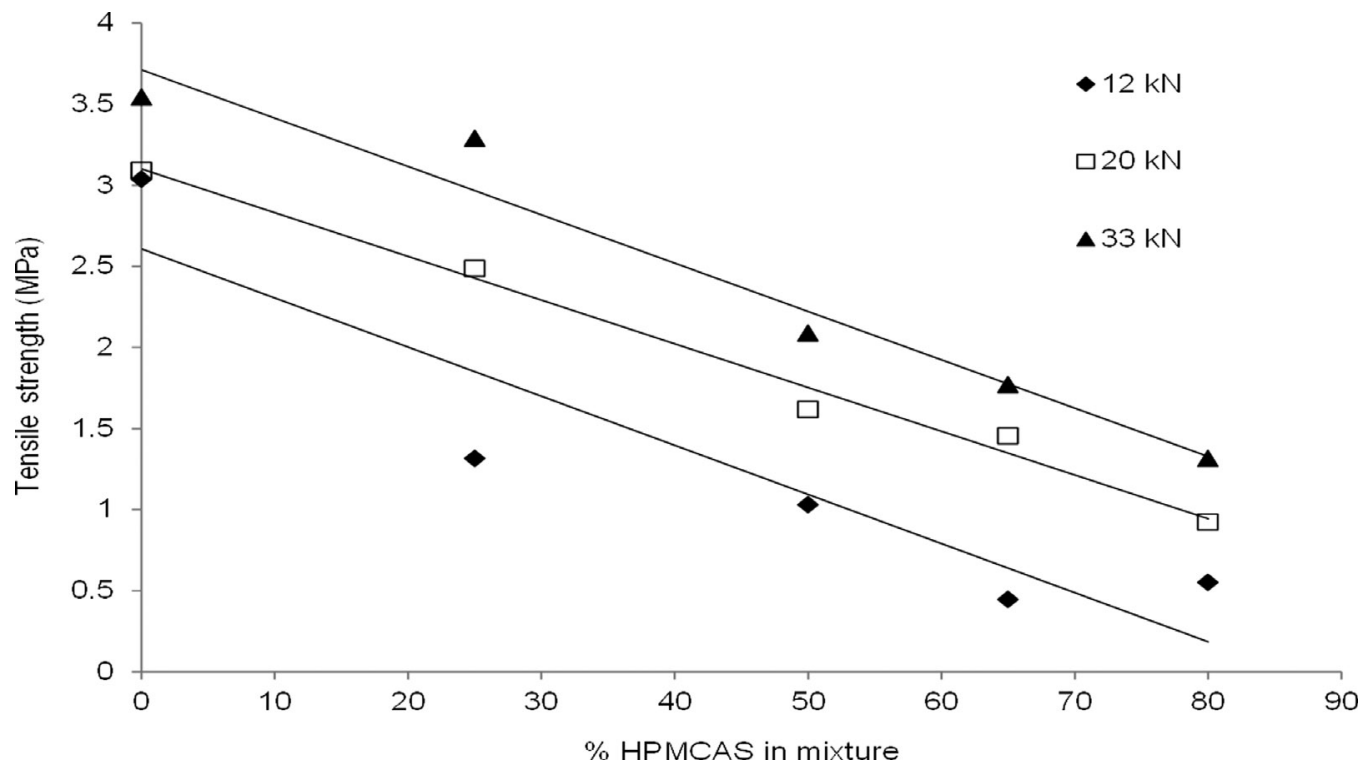

Figure 9. Tensile strength of tablets composed of mixtures of milled extrudate of HPMCAS and copovidone. 


\section{SUMMARY AND CONCLUSIONS}

The compaction properties (compactability, tabletability, compressibility) and Hiestand DTI were determined for a wide range of individual excipients in an "as is" and processed states. Furthermore, mixtures of excipients with and without an experimental drug Compound A in various states of processing beginning from melt extrusion to a final "compression ready" blend were prepared and evaluated for tableting indices. The overall analysis shows that the compaction properties and tableting indices can be effectively used to delineate and quantitate compression behavior of the excipients and differentiate the changes in their deformation behavior from "as is" to processed state. Importantly, the compaction properties and tableting indices of the materials tested indicate a composite behavior for mixtures containing excipients of different deformation that reflects the properties of the individual materials within the composite material. The bonding indices of poorly deforming materials were improved when plastically deforming materials were added, and this was supported by tableting studies. The DTI together with compaction properties (CP, SF, TS) thus provide an important and insightful "window" into the deformation attributes of different types of excipients. The changes in these attributes that occur as a result of processing can be quantitated, thus enabling the selection of excipients to compensate for material deficiencies during tablet development and develop formulations that can yield an acceptable tablet during powder compression.

\section{REFERENCES}

1. Hlinak AJ, Kuriyan K, Morris KR, Reklaitis GV, Basu PK. 2006. Understanding critical material properties for solid dosage form design. J Pharm Innovation 1(1):12-17.

2. Vachon MG, Chulia D. 1999. The use of energy indices in estimating powder compaction functionality of mixtures in pharmaceutical tableting. Int J Pharm 177(2):183-200.

3. Amidon GE. 1995. Physical and mechanical property characterization of powders. In Physical characterization of pharmaceutical solids; Brittain HG, Ed. Vol. 70. 1st ed. New York: Informa Healthcare, pp 281-317.

4. Amidon GE, Secreast PJ. 2009. Particle powder and compact characterization. In Pharmaceutical theory and practice in developing solid oral dosage forms; Qiu Y, Chen Y, Zhang GGZ, Lirong L, Porter WR, Eds. 1st ed. London: Academic Press, pp 163-183.

5. Jain S. 1999. Mechanical properties of powders for compaction and tableting: An overview. Pharm Sci Technol Today 2(1):20-31.

6. Final report on pharmaceutical cGMPs for 21st century-A risk-based approach. At: http://www.fda.gov/cder/gmp/gmp2004/GMP finalreport2004 Last accessed August 9, 2013.

7. Yu LX. 2008. Pharmaceutical quality by design: Product and process development, understanding, and control. Pharm Res 25(4):781-791.

8. Tye CK, Sun CC, Amidon GE. 2005. Evaluation of the effects of tableting speed on the relationships between compaction pressure, tablet tensile strength, and tablet solid fraction. J Pharm Sci 94:465472.

9. Hiestand EN, Smith DP. 1984. Three indices for characterizing the tableting performance of materials. Adv Ceram 9:47-57 (1984).

10. Hiestand EN, Smith DP. 1984. Indices of tableting performance. Powder Technol 38:145-159.

11. Hiestand EN. 1986. Tablet bond and related measurements. Pharm Tech 10:52-58.

12. Hiestand EN. 1989. The basis for practical applications of the tableting indices. Pharm Tech 9:54-66.
13. Hiestand EN. 1997. Mechanical properties of compacts and particles that control tableting success. J Pharm Sci 86:985990.

14. Venkatesh GM, Coleman JN, Wrzosek TJ, Duddu S, Palepu NR, Bandyopadhyay R, Grant DJW. 1998. Fractional factorial designs for optimizing experimental conditions for Hiestand's indices of tableting performance. Powder Technol 97(2):151-159.

15. Imbert C, Tchoreloff P, Leclerc B, Couarraze G. 1997. Indices of tableting performance and application of percolation theory to powder compaction. Eur J Pharm Biopharm 44(3):273-282.

16. Wurster DE, Likitlersuang S, Chen Y. 2005. The influence of magnesium stearate on the Hiestand tableting indices and other related mechanical properties of maltodextrins. Pharm Dev Technol 10(4):461466.

17. Uhumwangho MU, Okor RS, Eichie FE, Abbah CM. 2006. Influence of some starch binders on the brittle fracture tendency of paracetamol tablets. Afr J Biotechnol 5(20):1950-1953.

18. Sever NE, Ibrahim R, Sharma S, Schmidt C, Wu H, Haight A. 2003. Hiestand indices predict tablet compression behavior. AAPS Poster \# W4316. http://abstracts.aaps.org/published. to ALL]

19. Wurster DE, Majuru S, Oh E. 1999. Prediction of the Hiestand bonding indices of binary powder mixtures from single component bonding indices. Pharm Dev Technol 4(1):65-70.

20. Majuru S, Wurster DE. 1997. The effect of composition on the tableting indices of binary powder mixtures. Pharm Dev Technol 2(4):313321.

21. Kuppuswamy R, Anderson SR, Hoag SW, Augsburger LL. 2001. Practical limitations of tableting indices. Pharm Dev Technol 6(4):505520.

22. Amidon GE, Houghton ME. 1995. The effect of moisture on the mechanical and powder flow properties of microcrystalline cellulose. Pharm Res 12(6):923-929.

23. Crowley MM, Zhang F, Repka MA, Thumma S, Upadhye SB, Battu SK, McGinity JW, Martin C. 2007. Pharmaceutical applications of hotmelt extrusion: Part I. Drug Dev Ind Pharm. 33(9):909-926.

24. Repka MA, Battu SK, Upadhye SB, Thumma S, Crowley MM, Zhang F, Martin C, McGinity JW. 2007. Pharmaceutical applications of hot-melt extrusion: Part II. Drug Dev Ind Pharm. 33(10):10431057.

25. Chokshi RJ, Sandhu HK, Iyer RM, Shah NH, Malick AW, Zia H. 2005. Characterization of physico-mechanical properties of indomethacin and polymers to assess their suitability for hot-melt extrusion process as a means to manufacture solid dispersion/solution. J Pharm Sci 94(11):2463-2474.

26. Friesen DT, Shanker R, Crew M, Smithey DT, Curatolo WJ, Nightingale JAS. 2008. Hydroxypropyl methylcellulose acetate succinate-based spray-dried dispersions: An overview. Mol Pharm 5(6):1003-1019.

27. Janssens S, Mooter GV. 2009. Physical chemistry of solid dispersions. J Pharm Pharmacol 61:1571-1586.

28. Aharoni SM. 1998. Increased glass transition temperature in motionally constrained semicrystalline polymers. Polym Adv Technol 9(3):169-201.

29. Repka MA, Gerding TG, Repka SL, McGinity JW. 1999. Influence of plasticizers and drugs on the physical-mechanical properties of hydroxypropylcellulose films prepared by hot-melt extrusion. Drug Dev Ind Pharm 25(5):625-633.

30. Crowley MM, Fredersdorf A, Schroeder B, Ucera S, Prodduturi S, Repka MA. 2004. The influence of guaifenesin and ketoprofen on the properties of hot-melt extruded polyethylene oxide films. Eur J Pharm Sci 22(5):409-418.

31. Zhu Y, Shah NH, Malick AW, Infeld MH, McGinity JW. 2002. Influence of thermal processing on the properties of chlorpheniramine maleate tablets containing an acrylic polymer. Pharm Dev Technol 7(4):481-489.

32. Young CR, Dietzsch C, McGinity JW. 2005. Compression of controlled-release pellets produced by a hot-melt extrusion and spheronization process. Pharm Dev Technol 1:133-139. 
33. Crowley MM, Schroeder B, Fredersdorf A, Obara S, Talarico M, Kucera S. 2004. Physicochemical properties and mechanism of drug release from ethyl cellulose matrix tablets prepared by direct compression and hot-melt extrusion. Int J Pharm 271(1-2):77-84.

34. Carlson GT, Hancock BC. 2006. A comparison of physical and mechanical properties of common tableting diluents. In Excipient development for pharmaceutical, biotechnology, and drug delivery systems Katdare AV, Chaubal MV, Eds. 1st ed. New York: Informa Healthcare, pp 127-153.
35. He X, Secreast PJ, Amidon GE. 2007. Mechanistic study of the effect of roller compaction and lubricant on tablet mechanical strength. J Pharm Sci 96(5):1342-1355.

36. Ryskevitch E. 1953. Discussion of ball milling of pure ceramics diborides. J Am Ceram Soc 36:65-68.

37. Sarode A, Sandhu H, Iyer R, Shah N, Obara S, Tanno FK, Zia H. 2009. Stability assessment of hypromellose acetate succinate NF (HPMCAS) for application in hot melt extrusion. AAPS Poster \# T3368. http://abstracts.aaps.org/published. to ALL] 